\title{
An approximation method using approximate approximations
}

\author{
FRANK MÜLLER and WERNER VARNHORN, University of Kassel, Germany, \\ email: fmueller@mathematik.uni-kassel.de, varnhorn@mathematik.uni-kassel.de \\ (September 2005)
}

The aim of this paper is to extend the method of approximate approximations to boundary value problems. This method was introduced by V. Maz'ya in 1991 and has been used until now for the approximation of smooth functions defined on the whole space and for the approximation of volume potentials.

In the present paper we develop an approximation procedure for the solution of the interior Dirichlet problem for the Laplace equation in two dimensions using approximate approximations. The procedure is based on potential theoretical considerations in connection with a boundary integral equations method and consists of three approximation steps as follows.

In a first step the unknown source density in the potential representation of the solution is replaced by approximate approximations. In a second step the decay behavior of the generating functions is used to gain a suitable approximation for the potential kernel, and in a third step Nyström's method leads to a linear algebraic system for the approximate source density. For every step a convergence analysis is established and corresponding error estimates are given.

Keywords: Approximate Approximations, Laplace Equation, Dirichlet Problem, Boundary Integral Equation

2000 Mathematics Subject Classifications: 35J05, 45B05, 65N38

\section{Introduction}

In $1991 \mathrm{~V}$. Maz'ya proposed a new approximation method called the method of approximate approximations [8], which is based on generating functions representing an approximate partition of unity, only. As a consequence, this approximation method does not converge if the mesh size tends to zero.

For practical computations this lack of convergence does not play an important role since the resulting error can be chosen less than machine precision. On the other hand, this method has great advantages due to nice properties of the generating functions, i.e. simplicity, smoothness and exponential decay behavior [12].

The method of approximate approximations can be used efficiently for the evaluation of various problems in mathematical physics, e.g. Cauchy problems of the kind $L u=f$, where $L$ denotes a suitable linear differential operator in $\mathbb{R}^{n}$. Approximating the right hand side $f$ by approximate approximations, in many cases explicit formulas for the approximating volume potentials are 
obtained containing a one-dimensional integration, only.

For boundary value problems the method of approximate approximations has not been used until now, except for some heuristic considerations concerning the so-called boundary point method [9].

In the present paper we develop an approximation method for the solution of the interior Dirichlet problem of the Laplacian in two dimensions using approximate approximations. The procedure is based on potential theoretical considerations in connection with a boundary integral equations method and consists of three approximation steps as follows.

In a first step the unknown source density in the potential representation of the solution is replaced by approximate approximations. In a second step the decay behavior of the generating functions is used to gain a suitable approximation for the potential kernel, and in a third step Nyström's method leads to a linear algebraic system for the approximate source density. For every step a convergence analysis is established and corresponding error estimates are given.

The method, which is explicitly carried out here for the interior Dirichlet problem of the Laplacian in two dimensions, can also be used for many other boundary value problems in mathematical physics, whenever a suitable potential theory is available, as it is e.g. in the case of harmonic, elastic, or hydrodynamic boundary value problems in general space dimensions. In all these cases there exists also a representation of the solution in form of a boundary layer potential, where the unknown source density has to be determined as a solution of Fredholm boundary integral equations and can be approximated by the method developed here, too.

\section{The Dirichlet problem}

Let $G \subset \mathbb{R}^{2}$ be a bounded simply connected domain with boundary $\Gamma$ of class $C^{2}$. Let furthermore $b \in C(\Gamma)$ be a given boundary value. We consider the following Dirichlet problem: Find a function $v \in C^{2}(G) \cap C(\bar{G})$ solving

$$
\Delta v=0 \quad \text { in } G, \quad v=b \text { on } \Gamma \text {. }
$$

Here $\Delta$ denotes the Laplacian in $\mathbb{R}^{2}$. From potential theory it is well known $[2,4]$ that the Dirichlet problem has a unique solution $v$, and this solution can be represented in $G$ by the so called double layer potential

$$
(D \varphi)(x):=-\frac{1}{2 \pi} \int_{\Gamma} \frac{x-y}{|x-y|^{2}} \cdot n(y) \varphi(y) d s(y), \quad x \in G .
$$


The function $\varphi: \Gamma \rightarrow \mathbb{R}$ is an unknown density and $n(y)$ denotes the exterior unit normal vector in $y \in \Gamma$. It is known $[2,4]$ that under the regularity assumption $\Gamma \in C^{2}$ the kernel of the double layer potential can be continuously extended on $\Gamma \times \Gamma$. So for every $x \in \Gamma$ there exists the so-called direct value of the double layer potential

$$
(D \varphi)(x):=-\frac{1}{2 \pi} \int_{\Gamma} \frac{x-y}{|x-y|^{2}} \cdot n(y) \varphi(y) d s(y), \quad x \in \Gamma
$$

and defines a continuous function on $\Gamma$. Using the jump relations for the double layer potential $[2,4,5]$ it follows that the unknown density $\varphi$ exists as the unique solution to the Fredholm boundary integral equation of second kind

$$
\frac{1}{2} \psi(x)+(D \psi)(x)=b(x), \quad x \in \Gamma,
$$

where $(D \psi)(x)$ denotes the direct value of the double layer potential.

Let $\gamma:[-1,1] \rightarrow \Gamma$ be a parametrisation of the boundary $\Gamma$. Then we obtain both in $x \in G$ for the double layer potential and in $x \in \Gamma$ for its direct value the representation

$$
\begin{aligned}
(D \varphi)(x) & =-\frac{1}{2 \pi} \int_{-1}^{1} \frac{x-\gamma(t)}{|x-\gamma(t)|^{2}} \cdot n(\gamma(t))\left|\gamma^{\prime}(t)\right| \varphi(\gamma(t)) d t \\
& =-\frac{1}{2 \pi} \int_{-1}^{1} k(x, t)(\varphi \circ \gamma)(t) d t
\end{aligned}
$$

with

$$
k(x, t):=\frac{x-\gamma(t)}{|x-\gamma(t)|^{2}} \cdot n(\gamma(t))\left|\gamma^{\prime}(t)\right|
$$

Now we consider the operator

$$
T: C([-1,1]) \rightarrow C([-1,1])
$$

defined by

$$
(T \eta)(s):=\frac{1}{\pi} \int_{-1}^{1} k(\gamma(s), t) \eta(t) d t
$$


and set

$$
B:=2 b \circ \gamma
$$

Since $\varphi$ is the unique solution to the boundary integral equation (2), the function

$$
u:=\varphi \circ \gamma
$$

is the unique solution to the integral equation

$$
\eta-T \eta=B
$$

\section{The approximation procedure}

As mentioned above, the unique solution $v$ of the Dirichlet problem (1) in $G$ can be represented by the double layer potential

$$
v(x)=-\frac{1}{2 \pi} \int_{-1}^{1} k(x, t) u(t) d t, \quad x \in G .
$$

In the following we will approximate $v$ in $G$ by an explicit analytic expression containing no integrals. This will be done in three steps. To do so let $N \in \mathbb{N}$, $d>0, h:=1 / N$.

\subsection{The first approximation step}

In a first step we replace the unknown function $u$ in the integral representation (4) of $v$ by the approximate approximation

$$
u_{d, h}:[-1,1] \rightarrow \mathbb{R}, \quad u_{d, h}(t)=\frac{1}{\sqrt{\pi d}} \sum_{m=-N}^{N} u(m h) e^{-\frac{(t-m h)^{2}}{d h^{2}}},
$$

and define for $x \in G$

$$
\begin{aligned}
\Psi_{d, h}(x) & :=-\frac{1}{2 \pi} \int_{-1}^{1} k(x, t) u_{d, h}(t) d t \\
& =-\frac{1}{2 \pi \sqrt{\pi d}} \sum_{m=-N}^{N} u(m h) \int_{-1}^{1} k(x, t) e^{-\frac{(t-m h)^{2}}{d h^{2}}} d t
\end{aligned}
$$


as an approximation of $v(x)$.

\subsection{The second approximation step}

Since the function

$$
t \mapsto e^{-\frac{(t-m h)^{2}}{d h^{2}}}
$$

decreases rapidly outside of $m h$, if the term $d h^{2}$ is sufficiently small, in a second step we replace the kernel $k(x, t)$ by $k(x, m h)$ and define for $x \in G$

$$
\begin{aligned}
\Phi_{d, h}(x) & :=-\frac{1}{2 \pi \sqrt{\pi d}} \sum_{m=-N}^{N} u(m h) \int_{-1}^{1} k(x, m h) e^{-\frac{(t-m h)^{2}}{d h^{2}}} d t \\
& =-\frac{1}{2 \pi \sqrt{\pi d}} \sum_{m=-N}^{N} u(m h) k(x, m h) \int_{-1}^{1} e^{-\frac{(t-m h)^{2}}{d h^{2}}} d t
\end{aligned}
$$

as an approximation of $\Psi_{d, h}(x)$. Using

$$
\int_{-1}^{1} e^{-\frac{(t-m h)^{2}}{d h^{2}}} d t=\frac{\sqrt{d}}{N} \int_{\frac{m-N}{\sqrt{d}}}^{\frac{m+N}{\sqrt{d}}} e^{-t^{2}} d t=\frac{\sqrt{\pi d}}{2 N} \operatorname{erf}\left(\frac{m-N}{\sqrt{d}}, \frac{m+N}{\sqrt{d}}\right)
$$

we get

$$
\Phi_{d, h}(x)=-\frac{1}{4 \pi N} \sum_{m=-N}^{N} u(m h) k(x, m h) \operatorname{erf}\left(\frac{m-N}{\sqrt{d}}, \frac{m+N}{\sqrt{d}}\right),
$$

where for $a, b \in \mathbb{R}$ with $a \leq b$ the error function is defined by

$$
\operatorname{erf}(a, b):=\frac{2}{\sqrt{\pi}} \int_{a}^{b} e^{-t^{2}} d t
$$

\subsection{The third approximation step}

Since the density $\varphi$ is still unknown we do not know the values $u(m h)=$ $\varphi(\gamma(m h))$. Therefore in a third step we determine approximate values $u_{m}$ of $u(m h)$ by using Nyström's method [7]. Since $u=\varphi \circ \gamma$ is the unique solution of the integral equation (3) we have

$$
u(s)-(T u)(s)=B(s), \quad s \in[-1,1] .
$$


Hence, in particular,

$$
u(j h)-(T u)(j h)=B(j h), \quad j=-N, \ldots, N,
$$

which means

$$
u(j h)-\frac{1}{\pi} \int_{-1}^{1} k(\gamma(j h), t) u(t) d t=B(j h), \quad j=-N, \ldots, N
$$

Approximating the integral by the trapezoidal rule (Nyström's method) we get the linear system

$$
\sum_{m=-N}^{N}\left(\frac{\delta_{|m| N}-2}{2 \pi N} k(\gamma(j h), m h)+\delta_{j m}\right) u_{m}=B(j h), \quad j=-N, \ldots, N
$$

to determine approximate values $u_{m}$ of $u(m h)$. Here $\delta_{j m}$ denotes the Kronecker symbol. Setting

$$
a_{j m}:=\frac{\delta_{|m| N}-2}{2 \pi N} k(\gamma(j h), m h)+\delta_{j m}
$$

and

$$
A:=\left(a_{j m}\right),
$$

the linear system can now be written as follows:

$$
A\left(\begin{array}{c}
u_{-N} \\
\vdots \\
u_{N}
\end{array}\right)=\left(\begin{array}{c}
B(-1) \\
\vdots \\
B(1)
\end{array}\right)
$$

With help of its solution, for $x \in G$ we define

$$
v_{d, h}(x):=-\frac{1}{4 \pi N} \sum_{m=-N}^{N} u_{m} k(x, m h) \operatorname{erf}\left(\frac{m-N}{\sqrt{d}}, \frac{m+N}{\sqrt{d}}\right)
$$

as an approximation of $\Phi_{d, h}(x)$ and hence as an approximation of $v(x)$. 


\section{Convergence analysis}

To investigate the accuracy of the approximation, we define for $x \in G$ the error

$$
F(d, h, x):=\left|v(x)-v_{d, h}(x)\right|
$$

and consider the decomposition

$$
\begin{aligned}
F(d, h, x) & =\left|v(x)-\Psi_{d, h}(x)+\Psi_{d, h}(x)-\Phi_{d, h}(x)+\Phi_{d, h}(x)-v_{d, h}(x)\right| \\
& \leq \underbrace{\left|v(x)-\Psi_{d, h}(x)\right|}_{=: F_{1}(d, h, x)}+\underbrace{\left|\Psi_{d, h}(x)-\Phi_{d, h}(x)\right|}_{=: F_{2}(d, h, x)}+\underbrace{\left|\Phi_{d, h}(x)-v_{d, h}(x)\right|}_{=: F_{3}(d, h, x)},
\end{aligned}
$$

where $F_{i}(d, h, x)$ denotes the error in the $i$-th approximation step.

In the following we assume $b \in C^{2}(\Gamma)$. Then we find $\varphi \in C^{2}(\Gamma)$ due to the regularizing properties of the double layer potential [4]. Since also $\Gamma$ is of class $C^{2}$ we obtain $\gamma \in C^{2}\left([-1,1], \mathbb{R}^{2}\right)$ and hence $u \in C^{2}([-1,1])$.

For the convergence analysis below we need an estimate for the kernel. Using the Cauchy-Schwarz inequality we get

$$
|k(x, t)|=\left|\frac{x-\gamma(t)}{|x-\gamma(t)|^{2}} \cdot n(\gamma(t))\right| \gamma^{\prime}(t)|| \leq \frac{\left|\gamma^{\prime}(t)\right|}{|x-\gamma(t)|} \leq \frac{\left\|\gamma^{\prime}\right\|_{\infty}}{\operatorname{dist}(x, \Gamma)},
$$

where $\|\cdot\|_{\infty}$ denotes the supremum norm and

$$
\operatorname{dist}(x, \Gamma):=\inf _{y \in \Gamma}|x-y|
$$

the distance from $x \in G$ to the boundary $\Gamma$.

To prove convergence we begin from the back.

\subsection{Convergence in the third step}

It is known that Nyström's method converges in this setting of at least second order $[5,7]$. Hence for sufficently large $N$ the linear system (5) has a unique solution and there is a constant $C>0$ with

$$
\max _{m \in\{-N, \ldots, N\}}\left|u(m h)-u_{m}\right| \leq C h^{2} .
$$

This lead to an estimate for the corresponding error in the third step. 
Lemma 4.1 For $F_{3}(d, h, x)$ we have

$$
F_{3}(d, h, x) \leq \frac{3 C}{2 \pi} \frac{\left\|\gamma^{\prime}\right\|_{\infty}}{\operatorname{dist}(x, \Gamma)} h^{2}
$$

with the constant $C$ from (7).

Proof Using the estimates (6), (7) and

$$
\left|\operatorname{erf}\left(\frac{m-N}{\sqrt{d}}, \frac{m+N}{\sqrt{d}}\right)\right| \leq 2
$$

we obtain

$$
\begin{aligned}
F_{3}(d, h, x) & \leq \frac{1}{4 \pi N} \sum_{m=-N}^{N}\left|u(m h)-u_{m}\right||k(x, m h)| \operatorname{erf}\left(\frac{m-N}{\sqrt{d}}, \frac{m+N}{\sqrt{d}}\right) \\
& \leq \frac{1}{4 \pi N} \sum_{m=-N}^{N} C h^{2} \frac{\left\|\gamma^{\prime}\right\|_{\infty}}{\operatorname{dist}(x, \Gamma)} 2 \\
& =\frac{C}{2 \pi} \frac{\left\|\gamma^{\prime}\right\|_{\infty}}{\operatorname{dist}(x, \Gamma)} h^{3}(2 N+1) .
\end{aligned}
$$

With $2 N+1 \leq 3 N$ the assertion is proved.

\subsection{Convergence in the second step}

For $x \in G$ the function $t \mapsto k(x, t)$ is continuously differentiable in $[-1,1]$. So there exists

$$
L(x):=\max _{t \in[-1,1]}\left|\partial_{t} k(x, t)\right|
$$

wich is needed for the next estimate.

Lemma 4.2 For $F_{2}(d, h, x)$ we have

$$
F_{2}(d, h, x) \leq \frac{3\|u\|_{\infty} L(x)}{2 \pi^{3 / 2}} \sqrt{d} h .
$$

Proof Using a mean value theorem, for $t, m h \in[-1,1]$ we find

$$
|k(x, t)-k(x, m h)| \leq L(x)|t-m h| .
$$


Due to

$$
\begin{aligned}
\int_{-1}^{1}|t-m h| e^{-\frac{(t-m h)^{2}}{d h^{2}}} d t & =\frac{d}{N^{2}}\left(1-\frac{1}{2} e^{-\frac{1}{d}(m-N)^{2}}-\frac{1}{2} e^{-\frac{1}{d}(m+N)^{2}}\right) \\
& \leq \frac{d}{N^{2}}
\end{aligned}
$$

this implies

$$
\begin{aligned}
\int_{-1}^{1}|k(x, t)-k(x, m h)| e^{-\frac{(t-m h)^{2}}{d h^{2}}} d t & \leq L(x) \int_{-1}^{1}|t-m h| e^{-\frac{(t-m h)^{2}}{d h^{2}}} d t \\
& \leq \frac{d L(x)}{N^{2}}
\end{aligned}
$$

and hence

$$
\begin{aligned}
F_{2}(d, h, x) & \leq \frac{\|u\|_{\infty}}{2 \pi \sqrt{\pi d}} \sum_{m=-N}^{N} \int_{-1}^{1}|k(x, t)-k(x, m h)| e^{-\frac{(t-m h)^{2}}{d h^{2}}} d t \\
& \leq \frac{\|u\|_{\infty}}{2 \pi \sqrt{\pi d}} \sum_{m=-N}^{N} \frac{d L(x)}{N^{2}} \\
& =\frac{\|u\|_{\infty}}{2 \pi \sqrt{\pi d}} \frac{d L(x)(2 N+1)}{N^{2}}
\end{aligned}
$$

which completes the proof.

\subsection{Convergence in the first step}

For $m \in\{-N, \ldots, N\}$ we consider the linear spline

$$
s_{m}: \mathbb{R} \rightarrow \mathbb{R}, \quad s_{m}(t):=\left\{\begin{array}{cc}
1-N|t-m h|, t \in[m h-h, m h+h] \\
0 \quad, \quad \text { elsewhere }
\end{array}\right.
$$

and define the spline interpolant

$$
u_{h}^{s p}:[-1,1] \rightarrow \mathbb{R}, \quad u_{h}^{s p}(t):=\sum_{m=-N}^{N} u(m h) s_{m}(t)
$$


Since $u \in C^{2}([-1,1])$, it holds the estimate [7]

$$
\left\|u-u_{h}^{s p}\right\|_{\infty} \leq \frac{1}{8}\left\|u^{\prime \prime}\right\|_{\infty} h^{2} .
$$

Using the linear spline interpolant instead of approximate approximations in the first and second approximation step, we obtain

$$
\begin{aligned}
\Psi_{h}^{s p}(x) & :=-\frac{1}{2 \pi} \int_{-1}^{1} k(x, t) u_{h}^{s p}(t) d t \\
& =-\frac{1}{2 \pi} \sum_{m=-N}^{N} u(m h) \int_{-1}^{1} k(x, t) s_{m}(t) d t
\end{aligned}
$$

and

$$
\begin{aligned}
\Phi_{h}^{s p}(x) & :=-\frac{1}{2 \pi} \sum_{m=-N}^{N} u(m h) k(x, m h) \int_{-1}^{1} s_{m}(t) d t \\
& =-\frac{1}{4 \pi N} \sum_{m=-N}^{N} u(m h) k(x, m h)\left(2-\delta_{|m| N}\right)
\end{aligned}
$$

respectively.

The next lemma shows a remarkable convergence result if the parameter $d$ tends to zero.

Lemma 4.3 For all $x \in G$ we have

$$
\lim _{d \rightarrow 0} \Phi_{d, h}(x)=\Phi_{h}^{s p}(x) .
$$

Proof Using

$$
\lim _{d \rightarrow 0} \operatorname{erf}\left(\frac{m-N}{\sqrt{d}}, \frac{m+N}{\sqrt{d}}\right)=\lim _{d \rightarrow 0} \frac{2}{\sqrt{\pi}} \int_{\frac{m-N}{\sqrt{d}}}^{\frac{m+N}{\sqrt{d}}} e^{-t^{2}} d t
$$

and

$$
\frac{2}{\sqrt{\pi}} \int_{0}^{\infty} e^{-t^{2}} d t=1
$$


we get

$$
\lim _{d \rightarrow 0} \operatorname{erf}\left(\frac{m-N}{\sqrt{d}}, \frac{m+N}{\sqrt{d}}\right)=\left\{\begin{array}{l}
1, m=-N \\
2,|m| \neq N \\
1, m=N
\end{array}\right.
$$

hence

$$
\lim _{d \rightarrow 0} \operatorname{erf}\left(\frac{m-N}{\sqrt{d}}, \frac{m+N}{\sqrt{d}}\right)=2-\delta_{|m| N} .
$$

This implies

$$
\begin{aligned}
\lim _{d \rightarrow 0} \Phi_{d, h}(x) & =\lim _{d \rightarrow 0}\left(-\frac{1}{4 \pi N} \sum_{m=-N}^{N} u(m h) k(x, m h) \operatorname{erf}\left(\frac{m-N}{\sqrt{d}}, \frac{m+N}{\sqrt{d}}\right)\right) \\
& =-\frac{1}{4 \pi N} \sum_{m=-N}^{N} u(m h) k(x, m h) \lim _{d \rightarrow 0} \operatorname{erf}\left(\frac{m-N}{\sqrt{d}}, \frac{m+N}{\sqrt{d}}\right) \\
& =-\frac{1}{4 \pi N} \sum_{m=-N}^{N} u(m h) k(x, m h)\left(2-\delta_{|m| N}\right) \\
& =\Phi_{h}^{s p}(x),
\end{aligned}
$$

as asserted.

With help of the above convergence result, the remaining error in the first approximation step can be controlled, too.

Lemma 4.4 Let $x \in G$. For every $\varepsilon>0$ there exists some $d_{0}>0$ such that for all $d \leq d_{0}$ we have

$$
F_{1}(d, h, x) \leq \varepsilon+\frac{\|u\|_{\infty} L(x)}{2 \pi} h+\frac{3\|u\|_{\infty} L(x)}{2 \pi^{3 / 2}} \sqrt{d} h+\frac{\left\|\gamma^{\prime}\right\|_{\infty}\left\|u^{\prime \prime}\right\|_{\infty}}{8 \pi \operatorname{dist}(x, \Gamma)} h^{2} .
$$

Proof Choose $\varepsilon>0$. Due to Lemma 4.3 there exists some $d_{0}>0$ such that for all $d \leq d_{0}$ the estimate

$$
\left|\Phi_{h}^{s p}(x)-\Phi_{d, h}(x)\right| \leq \varepsilon
$$


holds. Using the inequatities (6) and (10) we get

$$
\begin{aligned}
\left|v(x)-\Psi_{h}^{s p}(x)\right| & =\frac{1}{2 \pi}\left|\int_{-1}^{1} k(x, t)\left(u(t)-u_{h}^{s p}(t)\right) d t\right| \\
& \leq \frac{1}{2 \pi} \int_{-1}^{1}|k(x, t)|\left|u(t)-u_{h}^{s p}(t)\right| d t . \\
& \leq \frac{\left\|\gamma^{\prime}\right\|_{\infty}\left\|u^{\prime \prime}\right\|_{\infty}}{8 \pi \operatorname{dist}(x, \Gamma)} h^{2} .
\end{aligned}
$$

Futhermore we find

$$
\begin{aligned}
\left|\Psi_{h}^{s p}(x)-\Phi_{h}^{s p}(x)\right| & \leq \frac{1}{2 \pi} \sum_{m=-N}^{N}|u(m h)| \int_{-1}^{1}|k(x, t)-k(x, m h)| s_{m}(t) d t \\
& \leq \frac{\|u\|_{\infty} L(x)}{2 \pi} \sum_{m=-N}^{N} \int_{-1}^{1}|t-m h| s_{m}(t) d t \\
& \leq \frac{\|u\|_{\infty} L(x)}{2 \pi} \sum_{m=-N}^{N} \frac{h^{2}}{3} . \\
& \leq \frac{\|u\|_{\infty} L(x)}{2 \pi} h,
\end{aligned}
$$

where $L(x)$ ist the constant defined in (9). Using the decomposition

$$
\begin{aligned}
F_{1}(d, h, x) \leq & \left|v(x)-\Psi_{h}^{s p}(x)\right|+\left|\Psi_{h}^{s p}(x)-\Phi_{h}^{s p}(x)\right| \\
& +\left|\Phi_{h}^{s p}(x)-\Phi_{d, h}(x)\right|+\left|\Phi_{d, h}(x)-\Psi_{d, h}(x)\right|
\end{aligned}
$$

and Lemma 4.2, we obtain the assertion.

Now collecting Lemmata 4.1, 4.2, and 4.4, the following main estimate is proved:

Theorem 4.5 Let $x \in G$. For every $\varepsilon>0$ there exists some $d_{0}>0$ such that for all $d \leq d_{0}$ we have

$$
\begin{aligned}
F(x, d, h) \leq & \varepsilon+\frac{\|u\|_{\infty} L(x)}{2 \pi} h+\frac{3\|u\|_{\infty} L(x)}{\pi^{3 / 2}} \sqrt{d} h \\
& +\frac{3 C\left\|\gamma^{\prime}\right\|_{\infty}}{2 \pi \operatorname{dist}(x, \Gamma)}\left(1+\frac{\left\|u^{\prime \prime}\right\|_{\infty}}{4}\right) h^{2},
\end{aligned}
$$


where $C$ is the constant from (7), and $L(x)$ is defined in (9).

Remark 1 Since $\varepsilon$ can be chosen less than machine precision, we call this approximation procedure pseudo convergent of first order as $h \rightarrow 0$.

Remark 2 Numerical examples with analytic boundaries and boundary values show that for $N=100$ the error in the third step is already less than machine precision.

Remark 3 Approximate approximations for whole space problems lead to a better accuracy if the parameter $d$ increases. Our method, developed for boundary values problems, is more accurate if the parameter $d$ is small.

\section{References}

[1] L.C. Evans, Partial Differential Equations, Graduate Studies in Mathematics Vol. 19, AMS, 1998.

[2] G.B. Folland, Introduction to Partial Differential Equations, Princeton University Press, 1995.

[3] D. Gilbarg, N.S. Trudinger, Elliptic Partial Differential Equations of Second Order, Springer, 1998.

[4] M.N. Günther, Die Potentialtheorie und ihre Anwendungen auf Grundaufgaben der mathematischen Physik, Verlagsgesellschaft, Leipzig, 1957.

[5] W. Hackbusch, Integralgleichungen, Teubner, 1989

[6] T. Ivanov, Boundary Layer Approximate Approximations and Cubature of Potentials in Domains, Linköping Studies in Science and Technology, Theses No. 516, Linköping University, 1997.

[7] R. Kress, Linear Integral Equations, Springer, 1989

[8] V. Maz'ya, A New Approximation Method and its Applications to the Calculation of Volume Potentials, Boundary Point Method, in: 3. DFG-Kolloquium des DFG-Forschungsschwerpunktes Randelementmethoden, 30.09.-05.10.1991.

[9] V. Maz'ya, Boundary Point Method, LITH-MATH-R-91-44, Dept. of Math., Linköping University, 1991.

[10] V. Maz'ya, Approximate Approximations, in: The Mathematics of Finite Elements and Applications. Highlights 1993, J.R. Whiteman (ed.), 77-104, Wiley, Chichester, 1994.

[11] V. Maz'ya, G. Schmidt, Approximate Approximations and the Cubature of Potentials, Atti Accad. Naz. Lincei Cl. Sci. Fis. Mat. Natur. Rend. Lincei (9) Mat. Appl. 6, 161-184, 1995.

[12] V. Maz'ya, G. Schmidt, On Approximate Approximations using Gaussian Kernels, IMA J. Num. Anal. 16, 13-29, 1996.

[13] V. Maz'ya, G. Schmidt, Construction of Basis Functions for High Order Approximate Approximations, in: Mathematical Aspects of Boundary Element Methods, M. Bonnet (ed) et al., Chapman Hall/CRC Res. Notes Math. 414, 191-202, 200.

[14] V. Maz'ya, G. Schmidt, On Quasi-Interpolation with Non-Uniformly Distributed Centers on Domains and Manifolds, J. Approx. Theory 110, 125-145, 2001.

[15] V. Maz'ya, G. Schmidt, W. Wendland, On the Computation of Multi-Dimensional Layer Harmonic Potentials via Approximate Approximations, CALCOLO 40, 33-53, 2003. 